\title{
GRANULOMATOSIS WITH POLYANGIITIS AFTER CYTOMEGALOVIRUS INFECTION: CASE REPORT
}

Leila Patrícia Muniz Veloso ${ }^{1, *}$, Fernanda Amond Castro1, Lilian Santuza Santos Porto ${ }^{1}$

1.Santa Casa de Belo Horizonte, Belo Horizonte (MG), Brazil.

*Corresponding author: leilapmuniz@hotmail.com

\section{BACKGROUND}

Autoimmune diseases are determined by a combination of predisposing genetic and environmental factors, resulting in chronic self-perpetuating inflammation. Disturbance in the balance between regulatory and effector $T$ cells and accumulation of effector $T$ cells in tissues favor chronic inflammation and loss of control of autoreactive T cells. Granulomatosis with polyangiitis (GPA), in turn, has a distinct cellular characteristic of imbalance in effector and regulatory T cells. A causal relationship between human cytomegalovirus (HCMV) and autoimmune diseases is suggested by the literature. The specific immunopathogenic mechanisms, until then studied, by which HCMV could contribute to the course of autoimmune disease are molecular mimicry, with its lytic replication in various tissues and lifelong latency after primary infection, in addition to expansion of CD4 + T cells CD28. We describe below a case of GPA diagnosis after HCMV infection.

\section{CASE REPORT}

Male, 80 years old, previously healthy, hospitalized for investigation of afternoon fever for two months, accompanied by weight loss of $15 \mathrm{~kg}$, asthenia, persistent mild abdominal pain and livedo in upper limbs. Through laboratory tests, imaging tests, myelogram, bone marrow biopsy, colonoscopy and blood cultures, myeloproliferative diseases, solid neoplasms and infectious diseases such as endocarditis, hepatitis, syphilis, Epstein-Barr and HIV were excluded, except HCMV, with anti-HCMV IgG and IgM positive. He also had positive antineutrophil cytoplasmic antibody (C-ANCA), randomly distributed pulmonary nodules, stage II renal failure with a 24-hour proteinuria of $554 \mathrm{mg}$. Hypothesizing a causal relationship, treatment with ganciclovir was started and a renal biopsy was carried out, which showed proliferative glomerulonephritis in proliferative phase, in the pauci-immune form type III, also closing the diagnosis for GPA.

\section{CONCLUSION}

The importance of increasing anti-HCMV IgM seropositivity in patients with GPA is still unclear. In data available in the literature, there are possible ways to explain the association with this and other autoimmune diseases, among them the hypothesis that $\mathrm{HCMV}$ infection may also play an active role in the initiation of ANCA-associated vasculitis. 